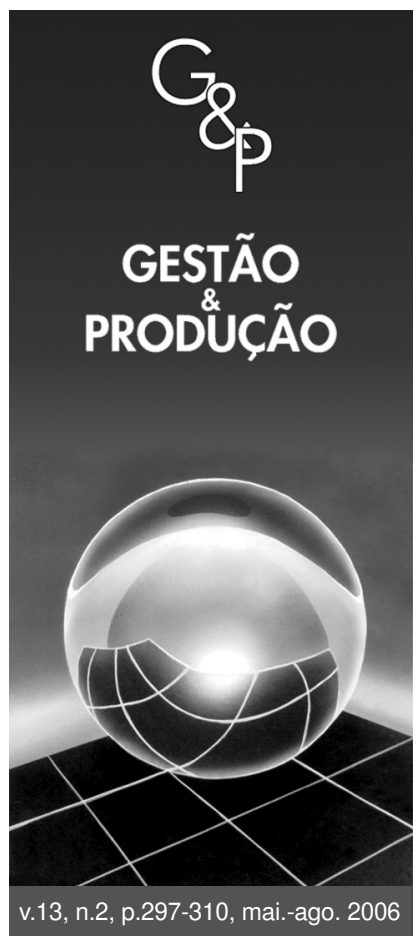

\title{
AVALIAÇÃO DA MATURIDADE DO PROCESSO DE DESENVOLVIMENTO DE VEÍCULOS AUTOMOTIVOS
}

\author{
Heitor Luiz Murat de Meirelles Quintella \\ Universidade Federal Fluminense, Engenharia de Produção, \\ Rua Passo da Pátria, 156, Sala 329, 24210-240, \\ São Domingos, Niterói, RJ, Brasil, \\ e-mail: hquintel@unisys.com.br \\ Henrique Martins Rocha \\ Associação Educacional Dom Bosco, \\ Estrada Resende Riachuelo, 2535, 27501-970, \\ Campo de Aviação, Resende, RJ, Brasil, \\ e-mail: hmartins@eng.aedb.br
}

Resumo

Neste estudo, buscou-se avaliar o nível de maturidade das organizações em seus processos de desenvolvimento de produtos, tendo como base os critérios do CMMI (Capability Maturity Model Integration). O referencial teórico utilizado foi o modelo de maturidade de capabilidade para desenvolvimento integrado de produtos e processos do SEI (Software Engineering Institute), da Carnegie Mellon University. Partindo da literatura existente sobre o assunto e de pesquisa e questionários aplicados em duas montadoras instaladas na região Sul Fluminense do País, identificou-se a existência de um processo estruturado de desenvolvimento de produtos, o qual é planejado, executado, medido e controlado e as práticas existentes são mantidas, mesmo nos momentos de crise, podendo repetir a experiência para novos projetos.

Palavras-chave: veículos automotivos, desenvolvimento de produtos, CMMI.

\section{Introdução}

O automóvel, produto que marcou o século XX como uma das expressões mais claras dos avanços tecnológicos, foi vetor de inovação com sua grande força difusora de novas tecnologias de produtos e de processos (Quintella et al., 2005a). Essas mudanças incluem novos materiais e soluções de engenharia, implicando em alterações nas estruturas de custos dos produtos e novas demandas para os fornecedores da cadeia automotiva. Conforme Alvarez et al. (2002), a fabricação automotiva foi responsável pela evolução dos sistemas de produção: as formas pelas quais se organizaram a produção e o trabalho humano passam necessariamente pelo eixo central do desenvolvimento dessa indústria, desde a introdução por Henry Ford das esteiras rolantes e postos de trabalho, com tarefas, partes e ferramentas repetitivas, dando início à produção em série (Quintella, 2000), passando pela produção enxuta e just in time da Toyota (Correa, 2004).
A cada ano, cerca de 60 milhões de veículos são produzidos no mundo (Oica, 2004), consumindo para isso mais de um trilhão de dólares e empregando cerca de quatro milhões de trabalhadores de produção direta e cerca de 16 milhões envolvidos em autopeças, vendas e serviços em todo o mundo (Arbix e Veiga, 2003). Responsável por $13,5 \%$ do PIB Industrial do Brasil (ANFAVEA, 2005), esta indústria coloca o país como o $12^{\circ}$ maior produtor de veículos do mundo e, ainda que com a ociosidade atual de quase $40 \%, 1,3$ milhão de empregos são gerados em toda a cadeia produtiva, em 200.000 empresas que atuam no setor - de montadoras a lojas de autopeças (Correa, 2004).

A vantagem competitiva de uma empresa na economia globalizada está diretamente relacionada com sua capacidade de introduzir novos produtos no mercado e que estes produtos venham a atrair a atenção dos clientes, os quais 
remuneram os investimentos feitos pela empresa (Rocha, 2005; Quintella e Rocha, 2006). No Brasil, encontramos exemplos de desenvolvimento de produtos pelas montadoras locais: modelos de sucesso de mercado como o EcoSport da Ford, Celta e Meriva da GM, Pálio da Fiat (parcialmente desenvolvido no Brasil) e o Fox da Volkswagen são exemplos desse movimento (Dias e Salerno, 2003; Sorima Neto e Grinbaum, 2004; Schibuola, 2004).

Uma das dificuldades encontradas pelas organizações no atual cenário competitivo é o gerenciamento integrado do processo de desenvolvimento de produtos, os quais devem ser tecnologicamente atualizados, com características de desempenho, custo e distribuição condizentes com o atual nível de exigência dos consumidores (Mundim et al., 2002). Shulman (2003) cita que "novos produtos são o motor número um do crescimento da renda", pois podem expandir a base de consumidores, atraindo novos clientes, ajudam a reter os atuais ao "continuamente atender a suas necessidades de mudança", aumentar a participação de mercado e a participação no negócio de cada cliente, proteger a companhia de avanços da competição, motivar empregados e partes envolvidas ao lidar com condições humanas que buscam a novidade.

Norton e Kaplan (1997) defendem a idéia de que, no desenvolvimento de produtos, é preferível que um novo produto ou a extensão de um produto antigo represente uma melhoria radical das opções atuais, que capturem novos clientes e mercados, e não se limitem a substituir as vendas dos produtos existentes. Tal conceito complementa a afirmação de Baxter (2003, p. 3) de que "deve-se estabelecer um compromisso entre os fatores que adicionam valor ao produto e aqueles que provocam aumento de custo". O autor defende que o planejamento estratégico do desenvolvimento do produto deve indicar quais são os produtos a serem desenvolvidos para atender aos objetivos da empresa, tratando-se, portanto, de um dos componentes do planejamento corporativo. Já Silva (2001) cita que, para ser fonte de competitividade, o "processo de desenvolvimento de produtos precisa ser eficiente e eficaz", sendo necessário a "utilização de metodologias e técnicas capazes de proporcionar tais atributos". Clark e Wheelwright (1992) defendem que, por essa razão, muitas organizações têm buscado os benefícios provenientes das novas tecnologias, visando a geração de ambientes industriais integrados e flexíveis, mais preparados para a realidade atual. No entanto, Silva (2001) destaca que "tais mudanças e os próprios produtos, cada vez mais complexos, vêm provocando alterações de natureza organizacional e tecnológica, acrescentando complexidade ao processo de desenvolvimento".

Gantewerker e Manoski (2003) expõem a importância estratégica contida no processo de desenvolvimento de produtos, principalmente nos estágios iniciais destes.
Segundo os autores, a direção estratégica da organização e as avaliações de viabilidade dos projetos são a base fundamental que trazem foco e clareza aos objetivos e a falha nesse ponto "cria uma fundação fraca para o projeto, levando normalmente a interações múltiplas e significativo retrabalho".

Clark e Fujimoto (1991) defendem que a estratégia de produto de uma empresa e como ela se organiza e gerencia o desenvolvimento, determinarão como o produto se portará no mercado: a maneira como esta realiza o desenvolvimento, sua velocidade, eficiência e qualidade do trabalho irão determinar a competitividade do produto. Da mesma forma, Clark e Wheelwright (1992) citam que, para este processo ser bem sucedido, deve conjugar velocidade no desenvolvimento com eficiência e eficácia. Esta abordagem mostra um paradoxo: como ter alta velocidade e baixo custo no desenvolvimento de produtos, conseguindo, no entanto, uma alta qualidade no processo em si e no produto gerado por meio dele? Baxter (2003) definiu regras básicas para o projeto sistemático:

- Estabelecimento de metas claras, concisas, específicas e verificáveis para o desenvolvimento de novos produtos;

- Acompanhamento do processo de geração do produto, comparando com as metas estabelecidas;

- Eliminação do produto que não esteja sendo desenvolvido no caminho adequado; e

- Geração de muitas idéias, para que possa ser escolhida a melhor.

Autores diversos (Hatch e Urban, 1974; Hisrich e Peters, 1978; Urban e Hauser; 1993, Gruenwald, 1994; Wilson et al., 1996; Semenik e Bamossy, 1996; Crawford, 1997; Kotler e Armstrong, 1999; Mattar e Santos, 2003) estabelecem etapas do processo de desenvolvimento de novos produtos que abordam em maior ou menor grau estes aspectos. Rozenfeld e Amaral (1999) estudaram modelos para desenvolvimento de produtos e identificaram fatores condicionantes nestes, tal como o grau de inovação do projeto, estrutura de mercado, direcionamento estratégico e dimensões da qualidade do produto. Segundo os autores, os fatores atuam de forma diferenciada no nível empresa, corporação e mercado. A tipologia defendida por estes encontra paralelo no modelo de fatores críticos de sucesso desenvolvido por Daniel (1961) e aperfeiçoado/ampliado por Rockart (1978; 1979). Já Costa Júnior e Silva (2003) estudaram os fatores de fracasso no processo de desenvolvimento de produtos, listados pelos autores como mau planejamento, mau gerenciamento, conceito ruim, má execução, mau uso da pesquisa e tecnologia ruim.

$\mathrm{Na}$ falta de um processo de desenvolvimento de produtos que abranja toda a organização, a repetição dos resultados depende inteiramente de se ter algumas pessoas disponíveis para o próximo projeto, o que não pro- 
vê bases para a melhoria da produtividade, qualidade e competitividade da organização por um longo período. Melhoramentos contínuos só podem ocorrer por esforços focados e sustentados na direção da construção de uma infra-estrutura de processo de efetivo desenvolvimento de produtos e práticas de gestão. Desta forma, faz-se necessário identificar o grau de maturidade dos processos de desenvolvimento de produtos no que tange à sua capacidade de serem definidos, utilizados, gerenciados, repetidos, que os resultados destes processos sejam previsíveis e possam ser continuamente melhorados, que é o objetivo deste estudo, por meio da seguinte questão: Qual o nível de maturidade dos métodos de desenvolvimento dos produtos utilizados pelas montadoras? Devido às restrições de tempo, custo e número de pessoas envolvidas, optou-se por delimitar a pesquisa às montadoras instaladas na região Sul Fluminense.

Conforme Rozenfeld et al. (2005), diferentes empresas adotam ferramentas e métodos distintos nos seus processos de desenvolvimento de produtos ou haverá usos diferenciados deles. Neste artigo, é apresentada a avaliação do nível de maturidade dos processos de desenvolvimento de produtos automotivos utilizados nas montadoras estudadas, tendo como base de avaliação os critérios do CMMI (Capability Maturity Model Integration).

\section{Referencial teórico}

\subsection{Modelos de maturidade}

O conceito de níveis de maturidade é baseado em princípios de qualidade de produto. A estrutura de maturidade, da qual esses princípios de qualidade foram adaptados, foi primeiramente mostrada por Crosby (1979) no chamado "Aferidor de Maturidade da Gerência de Qualidade", o qual descrevia cinco estágios na adoção das práticas de qualidade: Incerteza, Despertar, Esclarecimento, Sabedoria e Certeza. Esses princípios foram adaptados pelo SEI - Software Engineering Institute, da Carnegie Mellon University - para o processo de desenvolvimento de software em 1986 (Humphrey, 1987), pelo CMM (Capability Maturity Model). Outros modelos de maturidade foram desenvolvidos ao longo do tempo: o PMMM (Project Management Maturity Model), desenvolvido pelo Dr. Harold Kerzner (Kerzner, 2002), o OPM3 (Organizational Project Management Maturity Model), desenvolvido pelo Project Management Institute (PMI, 2003), o Project FRAMEWORK, da ESI International, o Project Management Maturity Assessment Model, da Knapp \& Moore Pty e, mais recentemente, o Modelo de Maturidade para PDP (Rozenfeld et al., 2005) e o PradoMMGP (Modelo de Maturidade em Gerenciamento de Projetos), desenvolvido pelo consultor brasileiro Darci Prado (Oliveira, 2006).
Além destes, de acordo com Sotille (2003), com base no sucesso do $C M M$, outros modelos foram criados, procurando cobrir outras áreas de interesse: o Software Acquisition CMM (SA-CMM), utilizado para avaliar a maturidade de uma organização em seus processos de seleção, compra e instalação de software desenvolvido por terceiros; o Systems Engineering CMM (SE-CMM), que avalia a maturidade da organização em seus processos de engenharia de sistemas, incluindo hardware, software e quaisquer outros elementos que participem do produto completo; o People $C M M(P-C M M)$, que avalia a maturidade da organização em seus processos de administração de recursos humanos, no que se refere a software: recrutamento e seleção de desenvolvedores, treinamento e desenvolvimento, remuneração, etc.; e, finalmente, o Integrated Product Development Capability Maturity Model (IPD-CMM), que inclui também os processos necessários à produção e suporte ao produto, tais como suporte ao usuário, processos de fabricação, etc. Este modelo serve de base para a melhoria de processos para todo o ciclo de vida do produto e para integração dos esforços de desenvolvimento de produtos por toda a organização. As melhores práticas do IPD-CMM foram incorporadas no CMMI - Capability Maturity Model Integration, sendo a fonte de IPD para a versão IPPD (Integrated Product and Process Development) do CMMI (Software Productivity Consortium, 2004), o qual foi utilizado para avaliação do nível de maturidade no processo de desenvolvimento de produtos neste estudo.

O projeto do CMMI (Integração dos Modelos de Maturidade de Capabilidade) foi desenvolvido para preservar os investimentos governamentais e da indústria em melhoria de processos e para substituir e melhorar os múltiplos modelos de maturidade que surgiram ao longo dos anos, além de facilitar o uso de tecnologia $C M M$ em diversas disciplinas, pelo uso de terminologias, componentes, métodos de avaliação e material de treinamento comuns (SEI, 2004). Este modelo, com crescente utilização no meio empresarial (Quintella et al., 2005b), foi motivo de estudo por Goldenson e Gibson (2003), que apresentaram os resultados da utilização do $C M M I$ em estudos de casos em diversas indústrias e organizações de diversos portes nos Estados Unidos, Europa e Austrália (Accenture Consulting, Boeing, Bosch, General Motors, JP Morgan Chase, Lockheed Martin, Northrop Grumman, Sanchez Computers, Thales Air Traffic, Training \& Simulation), com significativas melhorias de performance nas áreas de custo, prazos, qualidade, satisfação de clientes e retorno sobre investimentos.

\subsection{Componentes e estrutura do modelo CMMI}

O CMMI contém quatro áreas de conhecimento (disciplinas) em seu modelo: Engenharia de Sistemas (CMMI$S E)$, Engenharia de Software (CMMI-SW), Fonteamento 
de Fornecedores (CMMI-SS) e Desenvolvimento Integrado de Produto e Processo (CMMI-IPPD). O IPPD, utilizado nesta pesquisa, é uma abordagem sistemática que permite a colaboração ao longo do tempo das partes envolvidas (stakeholders) por toda a vida do produto, de forma a melhor satisfazer as necessidades, expectativas e requerimentos dos clientes, sendo seus processos integrados com os outros processos na organização (SEI, 2004). O CMMI fornece uma estrutura para organizar etapas evolutivas em cinco níveis de maturidade, que colocam fundamentos sucessivos para a contínua melhoria do processo, organizando as áreas de processos. Estas, por sua vez, contêm metas gerais e específicas, bem como práticas também genéricas e específicas. A Figura 1 a seguir mostra a estrutura e os componentes do modelo CMMI.

Nível de maturidade é um estágio evolutivo bem definido em direção à melhoria de processo, em que cada nível de maturidade fornece uma camada de fundamentos para a melhoria contínua do processo, provendo a forma de predizer a performance futura da organização em uma disciplina (ou conjunto de disciplinas). Cada nível compreende um conjunto de objetivos de processos que, quando satisfeitos, estabilizam componentes importantes de processo, resultando em um crescimento na capabilidade do processo da organização. Os níveis de maturida- de são designados por números, de 1 a 5 :

Nível de Maturidade 1: Inicial - a organização tipicamente não fornece um ambiente estável para desenvolvimento de processos, nem dispõe de práticas de gestão bem estabelecidas. Os benefícios das boas práticas de desenvolvimento de produtos são minados pelo planejamento ineficiente e por sistemas em que os compromissos são sempre reativos, ou seja, uma reação a algum acontecimento não planejado;

Nível de Maturidade 2: Gerenciado - todos os projetos da organização asseguram que os requerimentos, produtos e serviços são gerenciados e que os processos são planejados, executados, medidos e controlados. O status dos produtos e serviços é visível para a gerência em pontos específicos (milestones). A disciplina de processo ajuda a assegurar que as práticas existentes são mantidas durante os momentos de crise, com os projetos executados e gerenciados conforme os planos documentados. Compromissos são estabelecidos entre as partes interessadas (stakeholders) conforme a necessidade, sendo os produtos revistos por estes, para validação do atendimento de seus requerimentos, padrões e objetivos;

Nível de Maturidade 3: Definido - os processos são bem caracterizados e compreendidos, sendo descritos conforme padrões, procedimentos, ferramentas e mé-

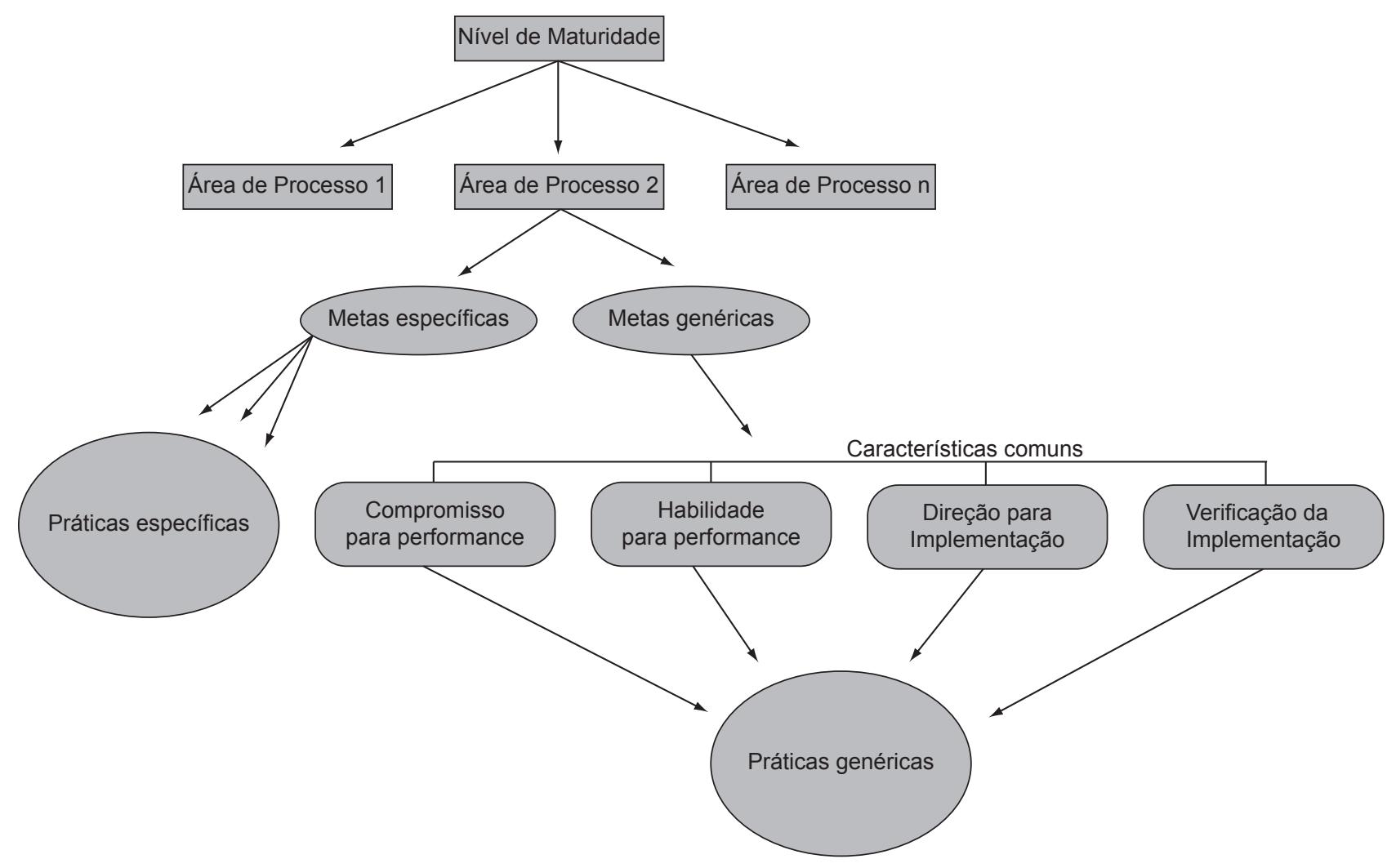

Figura 1. Componentes do Modelo CMMI. Fonte: SEI (2001a). 
todos. Os processos padrão de desenvolvimento e manutenção em toda a organização são documentados, incluindo padrões de gestão, sendo que esses processos são integrados de forma coerente. O conjunto de processos padrão, utilizados para estabelecer consistência ao longo de toda a organização, são estabelecidos e melhorados ao longo do tempo. A gerência da organização estabelece objetivos de processo baseados neste conjunto de processos padrão e assegura que esses objetivos são seguidos de forma apropriada. Um programa de treinamento é implementado para garantir que o pessoal e os gerentes tenham os conhecimentos e as habilidades requeridas para cumprir os papéis a eles designados;

Nível de Maturidade 4: Quantitativamente Gerenciado - a organização, além de estabelecer metas quantitativas de qualidade para os produtos e performance de processos, utiliza-as como critério de gerenciamento: os objetivos quantitativos são baseados nas necessidades dos clientes, usuários finais, implementadores de processo e a própria organização como um todo. A produtividade e a qualidade são medidas para as atividades importantes do processo de gerenciamento em todos os projetos, como parte de um programa organizacional de medidas, com os processos instrumentalizados com medições consistentes e bem definidas. Essas medições estabelecem a fundamentação para avaliar os processos e os produtos do projeto. Os projetos conseguem o controle sobre seus produtos e processos, reduzindo a variação no desempenho dos seus processos para cair em limites quantitativos aceitáveis, sendo compreendidos em termos estatísticos e gerenciados ao longo de toda a vida dos processos. Os riscos envolvidos na introdução de um novo domínio de aplicação são conhecidos e cuidadosamente gerenciados; e

Nível de Maturidade 5: Em Otimização - a organização inteira está focada na melhoria contínua da performance de processo, tanto por melhoria incremental (contínua) como por inovações tecnológicas. Objetivos mensuráveis de melhoria de processos são estabelecidos, continuamente revisados para refletir mudanças nos objetivos de negócio e utilizados como critério na melhoria do processo de gerenciamento. Os dados sobre a efetividade dos processos são usados para realizar análises de custo benefício das novas tecnologias e das mudanças propostas. Lições aprendidas são disseminadas para outros projetos e a otimização de processos velozes e inovativos depende da participação e empowerment da força de trabalho, alinhada com os objetivos e valores da organização e seus negócios. Melhoria de processos passa a ser parte da atividade de todos, levando a um ciclo de melhoria contínua.

Área de processo é um agrupamento de práticas relacionadas em uma área que, quando executadas coletivamente, satisfazem um grupo de metas consideradas importantes para significativas melhorias na área em questão. As áreas de processo são divididas em quatro categorias: Gerenciamento de Processos, Gerenciamento de Projetos, Engenharia e Suporte. O Quadro 1 a seguir mostra as áreas de processo correspondentes a cada nível de maturidade, servindo de base, portanto, para o enquadramento da organização no nível adequado.

Dentro dos diversos níveis de maturidade, são identificadas as metas específicas e genéricas, que devem ser

Quadro 1. Áreas de Processo por Nível de Maturidade. Fonte: elaboração própria.

\begin{tabular}{|c|c|c|c|c|c|}
\hline \multirow[t]{2}{*}{ Categorias } & \multirow[t]{2}{*}{ Áreas de Processo (PA's) } & \multicolumn{4}{|c|}{$\begin{array}{c}\text { Nível de } \\
\text { Maturidade } \\
\text { (ML) }\end{array}$} \\
\hline & & 2 & 3 & 4 & 5 \\
\hline \multirow[t]{5}{*}{$\begin{array}{l}\text { Gerenciamento } \\
\text { de Processos }\end{array}$} & $\begin{array}{l}\text { Foco no Processo } \\
\text { Organizacional }\end{array}$ & & $\mathrm{x}$ & $\mathrm{x}$ & $\mathrm{x}$ \\
\hline & $\begin{array}{l}\text { Definição do Processo } \\
\text { Organizacional }\end{array}$ & & $\mathrm{x}$ & $\mathrm{x}$ & $\mathrm{x}$ \\
\hline & Treinamento Organizacional & & $\mathrm{x}$ & $\mathrm{x}$ & $\mathrm{x}$ \\
\hline & $\begin{array}{l}\text { Performance de Processo } \\
\text { Organizacional }\end{array}$ & & & $\mathrm{x}$ & $\mathrm{x}$ \\
\hline & $\begin{array}{l}\text { Inovação e Desdobramento } \\
\text { Organizacional }\end{array}$ & & & & $\mathrm{x}$ \\
\hline \multirow{8}{*}{$\begin{array}{l}\text { Gerenciamento } \\
\text { de Projetos }\end{array}$} & Planejamento do Projeto & $\mathrm{x}$ & $\mathrm{x}$ & $\mathrm{x}$ & $\mathrm{x}$ \\
\hline & $\begin{array}{l}\text { Monitoramento e } \\
\text { Controle do Projeto }\end{array}$ & $\mathrm{x}$ & $\mathrm{x}$ & $\mathrm{x}$ & $\mathrm{X}$ \\
\hline & $\begin{array}{l}\text { Gerenciamento de Acordos } \\
\text { com Fornecedores }\end{array}$ & $\mathrm{x}$ & $\mathrm{x}$ & $\mathrm{x}$ & $\mathrm{x}$ \\
\hline & $\begin{array}{l}\text { Gerenciamento Integrado } \\
\text { do Projeto }\end{array}$ & & $\mathrm{x}$ & $\mathrm{x}$ & $\mathrm{x}$ \\
\hline & Gerenciamento de Riscos & & $\mathrm{x}$ & $\mathrm{x}$ & $\mathrm{x}$ \\
\hline & Integração do Time & & $\mathrm{x}$ & $\mathrm{x}$ & $\mathrm{x}$ \\
\hline & $\begin{array}{l}\text { Gerenciamento Integrado } \\
\text { de Fornecedores }\end{array}$ & & $\mathrm{x}$ & $\mathrm{x}$ & $\mathrm{x}$ \\
\hline & $\begin{array}{l}\text { Gerenciamento Quantitativo } \\
\text { do Projeto }\end{array}$ & & & $\mathrm{x}$ & $\mathrm{x}$ \\
\hline \multirow[t]{6}{*}{ Engenharia } & $\begin{array}{l}\text { Desenvolvimento dos Requeri- } \\
\text { mentos }\end{array}$ & & $\mathrm{x}$ & $\mathrm{x}$ & $\mathrm{x}$ \\
\hline & $\begin{array}{l}\text { Gerenciamento dos } \\
\text { Requerimentos }\end{array}$ & $\mathrm{x}$ & $\mathrm{x}$ & $\mathrm{x}$ & $\mathrm{x}$ \\
\hline & Solução Técnica & & $\mathrm{x}$ & $\mathrm{x}$ & $\mathrm{x}$ \\
\hline & Integração do Produto & & $\mathrm{x}$ & $\mathrm{x}$ & $\mathrm{x}$ \\
\hline & Verificação & & $\mathrm{x}$ & $\mathrm{x}$ & $\mathrm{x}$ \\
\hline & Validação & & $\mathrm{x}$ & $\mathrm{x}$ & $\mathrm{x}$ \\
\hline \multirow[t]{6}{*}{ Suporte } & Gerenciamento de Configuração & $\mathrm{x}$ & $\mathrm{x}$ & $\mathrm{x}$ & $\mathrm{x}$ \\
\hline & $\begin{array}{l}\text { Qualidade Assegurada de } \\
\text { Processo e Produto }\end{array}$ & $\mathrm{x}$ & $\mathrm{x}$ & $\mathrm{x}$ & $\mathrm{x}$ \\
\hline & Medição e Análise & $\mathrm{x}$ & $\mathrm{x}$ & $\mathrm{x}$ & $\mathrm{x}$ \\
\hline & $\begin{array}{l}\text { Ambiente Organizacional para } \\
\text { Integração }\end{array}$ & & $\mathrm{x}$ & $\mathrm{x}$ & $\mathrm{x}$ \\
\hline & Análise de Decisão e Resolução & & $\mathrm{x}$ & $\mathrm{x}$ & $\mathrm{x}$ \\
\hline & Análise Causal e Resolução & & & & $\mathrm{x}$ \\
\hline
\end{tabular}


alcançadas pelos processos de planejamento e implementação da organização, sendo essenciais para o atingimento dos níveis de maturidade. A satisfação das metas é utilizada em avaliações, como base para atendimento das áreas de processo e determinação do nível de maturidade da organização: uma área de processo é avaliada como "satisfeita" somente se todas as suas metas genéricas e metas específicas forem avaliadas como "satisfeitas". Se ao menos uma das metas for avaliada como "não-satisfeita", a área de processo como um todo será considerada "não satisfeita" (SEI, 2001b).

Para a realização da pesquisa, foi adotada a premissa de que a metodologia do $C M M I$ é válida para mensuração do nível de maturidade do processo de desenvolvimento de produtos das montadoras.

\section{Metodologia}

No presente trabalho, foi adotado o método hipotético-dedutivo, no qual a percepção de uma lacuna nos conhecimentos, acerca da qual se formulam as hipóteses, é seguida pelo processo de inferência dedutiva, testando-se a predição da ocorrência de fenômenos abrangidos pela hipótese. Desta forma, foi desenvolvida uma hipótese como solução provisória para o problema, a qual foi testada por meio da coleta de informações em pesquisa de campo e análise estatística dos resultados obtidos.

\subsection{Hipótese e questões-chave}

Hipótese I - Em busca do posicionamento estratégico e manutenção de sua competitividade, as empresas da indústria automotiva mantêm um método estruturado para desenvolvimento de produtos, o qual tem a potencialidade de garantir a aceitação dos consumidores e o fluxo financeiro advindo do sucesso comercial dos produtos.

Questões-chave:

1) O processo de desenvolvimento de produto é planejado, executado, medido e controlado e as práticas existentes são mantidas, mesmo nos momentos de crise, podendo repetir a experiência para novos projetos? (equivalente ao Nível 2 do $C M M I$ );

2) A organização possui um processo de desenvolvimento de produtos bem caracterizado e compreendido, sendo descrito na forma de padrões, procedimentos, ferramentas e métodos, com objetivos baseados nestes e utilizado em todos os projetos de desenvolvimento de produtos? (equivalente ao Nível 3 do $C M M I$ );

3) O processo de desenvolvimento de produtos da organização, já padronizado, é medido e opera dentro dos limites estabelecidos? (equivalente ao Nível 4 do CMMI); $\mathrm{e}$

4) A organização trabalha com inovação do processo de desenvolvimento de produtos, identificando as oportu- nidades de aperfeiçoamento, fortalecendo o processo de maneira pró-ativa e objetivando a prevenção de falhas? (equivalente ao Nível 5 do $C M M I$ ).

O quadro de amarração lógica das questões e de suas justificativas por nível de maturidade pode ser observado no Apêndice. Para determinação do nível de maturidade da organização, foram utilizadas 27 perguntas para avaliar a percepção dos entrevistados quanto ao atendimento ou satisfação das metas (genéricas e específicas) das áreas de processo, com base nos conceitos do CMMI. Tais perguntas buscaram avaliar o atingimento das metas nas áreas de processo dos níveis de maturidade em uma escala somatória (escala Likert). Proposta por Rensis Likert em 1932 (Mattar, 1996), esta compreende uma série de afirmações relacionadas com o objeto pesquisado, para determinação pelos entrevistados do seu grau de concordância/discordância das afirmações. A cada célula de resposta é atribuído um número que reflete a direção da atitude do respondente em relação a cada afirmação e a pontuação total da atitude de cada respondente é dada pela somatória das pontuações obtidas para cada afirmação. No questionário utilizado, adotou-se a seguinte escala: 1) Não/Nunca; 2) Raramente; 3) Às vezes; 4) Freqüentemente; e 5) Sempre.

\subsection{Teste da hipótese}

No teste da hipótese foram adotados os seguintes critérios:

1) O projeto pertencerá ao maior nível cuja mediana obtida pelas questões do CMMI for maior ou igual a quatro, desde que todas as respostas do grupo atinjam também valores iguais ou superiores a quatro. A razão para adotar-se tal valor como limite mínimo para identificação do nível de maturidade deve-se ao fato de que, utilizando-se os critérios do $C M M I$, a determinação do nível de maturidade passa pelo atingimento ou satisfação das metas das áreas de processo: se qualquer meta for avaliada como "não-satisfeita", a área de processo será avaliada como "não-satisfeita" (SEI, 2001b). A representação em estágios dos níveis de maturidade do CMMI, não permite os "saltos" dos níveis, visto que os processos "não podem alcançar seus potenciais totais, até que a base adequada seja estabelecida" (SEI, 2001a, p. 15) e, sem essa base adequada, tendem a falhar em condições de estresse, não dando sustentação à melhoria contínua dos processos. Desta forma, o escore três, entendido como "às vezes" no questionário, não representaria fielmente o atingimento, optando-se, portanto, pelo escore quatro ("freqüientemente") como característico do atingimento destas; e

2) Qualquer resposta com mediana inferior a quatro, dentro de um grupo de perguntas, será interpretado como a não satisfação das metas para o nível, mesmo que a mediana do grupo seja igual ou superior a quatro; e 
3) O não atingimento do nível 2 de maturidade nas questões do $C M M I$ indicará que a organização se encontra no nível inicial (nível 1 de maturidade).

\subsection{Coleta de dados, populaçãol universo e amostra}

A coleta de dados nesta pesquisa foi realizada por meio da aplicação de questionário autopreenchido, ou seja, o questionário lido e preenchido diretamente pelos pesquisados. Os questionários - compostos de 27 perguntas que buscavam identificar a adoção de práticas estabelecidas pelo modelo do CMMI, de acordo com o nível de maturidade da organização - foram enviados e recebidos, após contato prévio, no qual foi solicitada a colaboração no desenvolvimento da pesquisa por meio do preenchimento do questionário por e-mail. Todos os participantes foram informados sobre a característica científica e acadêmica da pesquisa e sobre seu propósito.

Conforme citado anteriormente, optou-se por delimitar a pesquisa às montadoras instaladas na região Sul Fluminense, ou seja, uma empresa voltada para o segmento de automóveis e outra para o segmento de caminhões e ônibus. Os questionários foram direcionados a gerentes, supervisores e corpo técnico de Marketing/Comercial, Produto, Logística, Suprimentos e Manufatura das montadoras. Tais questionários eram compostos de blocos de perguntas, que buscavam determinar o nível de maturidade da organização, avaliando a percepção dos entrevistados quanto ao atendimento ou satisfação das metas (genéricas e específicas) das áreas de processo dos níveis de maturidade, com base nos conceitos do CMMI. Foram respondidos 47 questionários, distribuídos conforme mostrado na Tabela 1 a seguir.

\subsection{Tratamento e análise dos dados}

Na pesquisa, como não havia conhecimento sobre os parâmetros da população, a escolha do teste estatístico para validação das hipóteses recaiu sobre os métodos não paramétricos (Mattar, 1996), utilizando os dados coletados na escala ordinal. Conforme diversos autores (Siegel, 1981; Hoel, 1984; Levin, 1987; Mattar, 1996; Cooper e Schindler, 2003), como os números nessas escalas têm

Tabela 1. Resumo dos respondentes.

\begin{tabular}{lrrrr}
\hline \multicolumn{1}{c}{ Área } & $\begin{array}{c}\text { Distribuição dos respondentes } \\
\text { Automóveis }\end{array}$ & $\begin{array}{c}\text { Caminhões e } \\
\text { ônibus }\end{array}$ \\
\hline Marketing/Comercial & 2 & $9 \%$ & 2 & $8 \%$ \\
Produto & 5 & $22 \%$ & 8 & $33 \%$ \\
Logística e Suprimentos & 8 & $35 \%$ & 7 & $29 \%$ \\
Manufatura e apoio & 8 & $35 \%$ & 7 & $29 \%$ \\
Total & 23 & $100 \%$ & 24 & $100 \%$ \\
\hline
\end{tabular}

Fonte: elaboração própria. significado apenas de classificação, a mensuração apropriada da tendência central é a mediana, enquanto a dispersão é revelada por medidas como percentuais ou quartis. A escolha do tipo de teste a ser aplicado à situação levou à seleção do teste de sinais para verificação da mediana dos resultados.

Os resultados (medianas) encontrados para cada questão em cada uma das organizações (AU - automóveis; e CA - caminhões e ônibus) podem ser observados na Tabela 2 a seguir.

\subsection{Limitações do método}

Há a possibilidade de respostas distorcidas, causadas pelo grau de motivação do entrevistado, preocupação em não transmitir uma avaliação ruim de si próprio ou de suas empresas, a falta de conhecimentos sobre o assunto pesquisado, assim como, a inadequação do questionário (excessivo número de perguntas, escala utilizada e tempo, entre outros). Buscou-se reduzir as potenciais distorções no contato anterior ao envio e preenchimento do questionário, quando se ressaltou a importância da imparcialidade e do sigilo da pesquisa. Nesta oportunidade, testou-se também a confiança do respondente quanto ao fato de estar capacitado a prover as respostas/avaliações solicitadas no questionário. Procurou-se evitar exercer qualquer influência sobre os respondentes, de modo a não influenciar suas respostas e efetuou-se pré-teste do questionário (quatro integrantes do universo pesquisado), buscando-se aperfeiçoá-lo e reduzir as falhas de entendimento.

\section{Análise e discussão dos resultados}

Os dados foram tabulados com a contagem da freqüência das notas atribuídas nas questões referentes aos aspectos de cada nível de maturidade, consolidando os resultados, quando se buscou identificar o nível de maturidade da organização no seu processo de desenvolvimento de produtos, tendo como base os critérios do $C M M I$, conforme mostrado nas Tabelas 3 e 4 a seguir, para cada organização:

Com base no critério de atingimento do escore mínimo de quatro (4) para identificação do nível de maturidade - por grupo de questões e por questão individual -, percebe-se que ambas as organizações alcançaram o nível de maturidade 2 - Gerenciado - para seu processo de desenvolvimento de produtos, pelos critérios do CMMI. Ainda que escores "4" fossem identificados em níveis superiores de maturidade nas organizações, o não atingimento deste escore em todas as perguntas no nível de maturidade 3 (vide coluna "Menor mediana"), caracteriza a não satisfação das metas das áreas de processo no nível de maturidade em questão, conforme citado anteriormente.

Não é possível fazer inferências envolvendo todos os níveis de maturidade, visto tratar-se de uma escala ordi- 


\section{Tabela 2. Resultados (medianas) por questão.}

\section{Questões}

1. Os projetos para desenvolvimento de produtos são planejados, com envolvimento das partes interessadas (inclusive fornecedores) e tais projetos são monitorados e controlados contra seu plano?

2. São estabelecidas ações corretivas quando o projeto de desenvolvimento não está acompanhando o plano para ele traçado?

3. Os requerimentos para o pwrojeto são coletados, mantidos atualizados e podem ser acessados, provendo rastreabilidade desde o cliente até o produto final e seus componentes?

4. Os planos, descrições de processos, requerimentos, informações de projeto, desenhos, especificações, dados de produto, etc., necessários ao desenvolvimento do produto, são mantidos, identificados, controlados e auditados?

5. Os processos, seus subprodutos e serviços no desenvolvimento dos produtos têm a performance avaliada objetivamente quanto a sua aderência às descrições de processos, objetivos e padrões durante toda a vida do projeto e as informações são passadas ao time do projeto, permitindo a visibilidade e acompanhamento por parte destes?

6. Todos os projetos da organização asseguram que os requerimentos, produtos e serviços são gerenciados e que os processos são planejados, executados, medidos e controlados, com o status dos produtos e serviços, sendo visíveis para a gerência em pontos específicos (milestones)?

7. As atividades, status e resultados de processos são revistos com a Alta Gerência?

8. São coletadas as informações, medidas e resultados de planejamento e execução do processo de desenvolvimento, de forma a entender as forças e fraquezas dos processos utilizados, planejar e implementar melhorias contínuas neles?

9. São estabelecidos e mantidos processos padrões da organização, os quais são baseados nas necessidades e objetivos desta, incluindo aí, além das descrições dos processos, o detalhamento de seus elementos, guias de orientação e documentação?

10. São identificadas as necessidades estratégicas de treinamento para a organização, bem como as necessidades táticas que são comuns entre projetos e grupos de suporte, obtendo ou desenvolvendo as habilidades requeridas para executar os processos padrões da organização?

11. Os envolvidos no desenvolvimento do novo produto têm uma visão compartilhada do projeto e coordenam seus esforços, envolvendo-se, identificando, negociando e acompanhando as interdependências existentes, de forma a resolver os problemas que surgem?

12. Essa integração e colaboração entre envolvidos no desenvolvimento de novos produtos são incentivadas, reconhecidas e/ou premiadas, promovendo a excelência de indivíduos e times?

13. Os riscos de desenvolvimento associados a custo, recursos, cronograma e aspectos técnicos do projeto são identificados, avaliados, documentados e as possíveis respostas a estas situações, identificadas?

14. As necessidades do cliente, depois de identificadas, são traduzidas em requerimentos de produto e, destes, identificados os requerimentos dos componentes do produto e, conseqüentemente, a performance esperada para cada um deles?

15. Tais requerimentos são convertidos na arquitetura do produto e projeto de componentes, com soluções que são analisadas com base em critérios formais de avaliação, que dependem do tipo de produto, requerimentos de performance, custos e prazos para desenvolvimento?

16. Assegura-se que o produto desenvolvido atende a seus requerimentos, partindo-se inicialmente da verificação dos seus componentes e processos e concluindo-se com a verificação do produto final?

17. O cliente é envolvido nesse processo de verificação, validando-o?

18. Os processos são bem caracterizados e compreendidos, sendo descritos conforme padrões, procedimentos, ferramentas e métodos e os processos padrões de desenvolvimento e manutenção em toda a organização são documentados, incluindo padrões de gestão, sendo que esses processos são integrados em um todo coerente?

19. Existe um programa de treinamento para garantir que o pessoal envolvido com o desenvolvimento e os gerentes tenham os conhecimentos e as habilidades requeridas para cumprir os papéis a eles designados?

20. Os objetivos de negócio da organização são desdobrados em objetivos quantitativos para qualidade e performance de processos, provendo os grupos de apoio e de projeto com indicadores, guias de orientação e modelos de performance de processos?

21. Tais objetivos quantitativos são baseados nas necessidades dos clientes, usuários finais, implementadores de processo e a própria organização como um todo?

22. Para estes objetivos quantitativos, são aplicadas técnicas de gerenciamento de performance de processo e produto que sejam quantitativas e estatísticas?

23. As variações significativas no desempenho dos processos podem ser distinguidas das variações aleatórias (ruídos), particularmente dentro de linhas de produtos estabelecidas e a causa raiz das variações é identificada e, quando apropriado, são corrigidas de forma a prevenir ocorrências futuras?

24. Os riscos envolvidos na introdução de um novo produto, tecnologia ou área de atuação ou aplicação são conhecidos e cuidadosamente gerenciados?

25. As propostas de melhoria inovativas e incrementais para melhoria da habilidade da organização em alcançar os objetivos de qualidade e performance de processos são selecionadas com base no entendimento quantitativo dos benefícios potenciais e custo de desenvolvimento, além da disponibilidade para investimento, envolvendo um grupo com poder de decisão e alinhado com os valores e objetivos da organização?

26. Busca-se entender as causas comuns de variação inerente aos processos e obter formas de removê-las destes processos, utilizando-se esse conhecimento e lições aprendidas para melhorar continuamente os processos da organização, disseminando-os também para outros projetos?

27. A organização inteira está focada na melhoria contínua da performance de processo, tanto por melhoria incremental (contínua) como por inovações tecnológicas (incluindo prevenção de falhas) e os objetivos mensuráveis de melhoria de processos são estabelecidos, continuamente revisados para refletir mudanças nos objetivos de negócio e utilizados como critério na melhoria do processo de gerenciamento?

Fonte: elaboração própria.

\begin{tabular}{cc}
\multicolumn{2}{c}{ Mediana } \\
AU & CA \\
\hline 4 & 4 \\
4 & 4 \\
4 & 4 \\
5 & 4 \\
4 & 4
\end{tabular}


Tabela 3. Resultados - empresa do segmento automóveis.

\begin{tabular}{cccccc}
\hline & \multicolumn{5}{c}{ Tabulação dos dados da questão } \\
Nível de Maturidade & Mediana & Desvio Médio & Mínimo & Máximo & Menor mediana \\
\hline 2 & 4 & 0,665 & 2 & 5 & 4 \\
3 & 4 & 0,668 & 1 & 5 & 3 \\
4 & 4 & 0,613 & 1 & 5 & 4 \\
5 & 4 & 0,661 & 2 & 5 & 4 \\
\hline
\end{tabular}

Fonte: elaboração própria.

Tabela 4. Resultados - empresa do segmento caminhões e ônibus.

\begin{tabular}{|c|c|c|c|c|c|}
\hline \multicolumn{6}{|c|}{ Tabulação dos dados da questão } \\
\hline Nível de Maturidade & Mediana & Desvio Médio & Mínimo & Máximo & Menor mediana \\
\hline 2 & 4 & 0,643 & 1 & 5 & 4 \\
\hline 3 & 4 & 0,707 & 1 & 5 & 3 \\
\hline 4 & 4 & 0,687 & 1 & 5 & 4 \\
\hline 5 & 4 & 0,699 & 1 & 5 & 4 \\
\hline
\end{tabular}

Fonte: elaboração própria.

nal. No entanto, dentro do nível de maturidade identificado para cada uma das organizações, foi possível avaliar a tendência central e a dispersão, com base nas pontuações compiladas dos respondentes, conforme apresentado na Tabela 5 a seguir.

Para se avaliar a confiança no nível de maturidade identificado, faz-se necessário estabelecer o grau de confiança no atingimento do nível mínimo de pontuação para o enquadramento no nível específico (quatro): conforme Triola (1999, p. 321), "podemos aplicar o teste de sinais a uma afirmação sobre a mediana de uma única população", enquanto Hoel (1984) cita que é possível testar valores de mediana pelo teste do sinal, restringindo, no entanto, a variáveis contínuas. Como Siegel (1981, p. 20) admite que numa escala ordinal, com suas categorias discretas, há um "continuum" de resultados possíveis - ainda que, por conta da "falta de precisão do aparelhamento de mensuração", não se distingam as pequenas diferenças que realmente existem entre as observações, o que resulta em menor poder-eficiência, usual em testes não paramétricos - utilizou-se tal teste na presente pesquisa. Conforme Siegel (1981), a distribuição amostral - para prova de sinais - tem distribuição aproximadamente normal para $\mathrm{N}>25$, permitindo o uso da estatística " $\mathrm{z}$ " com correção de continuidades (Triola, 1999), conforme a fórmula apresentada a seguir:

$$
z=\frac{(x+0,5)-(n / 2)}{n^{1 / 2} / 2}
$$

O levantamento das respostas às questões referentes ao nível de maturidade 2 apresentou os resultados mostrados na Tabela 6 a seguir.

Como o número de vezes que o sinal menos freqüente ocorre (x) correspondeu à expectativa inicial, da mediana ser inferior a quatro (uma vez eliminados os valores zero), deu-se prosseguimento ao teste: o calculo de "z", com base nos valores de "x" e "n" para as organizações, resultou em $-2,53$ e $-3,44$, para a empresa de automóveis e para a empresa de caminhões e ônibus, respectivamente. Utilizando-se a função "INV.NORMP" do MS Excel, foi possível calcular o valor do afastamento correspondente à probabilidade de $5 \%(0,05)$ em uma distribuição normal com média zero e desvio padrão 1, para o qual foi encontrado o valor - 1,645. Desta forma, concluiu-se que, sendo o " $z$ " de ambas as empresas mais afastado do valor central que o " $z$ " crítico, com base na evidência amostral, em um nível de significância de $\alpha=0,05$, ambas as organizações têm uma mediana igual ou maior que quatro nas perguntas referentes ao nível de maturidade 2, conforme pode ser observado na Figura 2 a seguir.

\subsection{Discussão dos resultados}

$\mathrm{O}$ estudo mostrou ambas as organizações atingindo o nível 2 de maturidade de desenvolvimento de produtos - característica de um processo dito "gerenciado" - com base nos referenciais do CMMI e no critério da obtenção da pontuação mínima " 4 " - equivalente às práticas sendo freqüentemente utilizadas - nas perguntas referentes aos requisitos para o nível de maturidade em questão. Apesar de ambas as organizações alcançarem a mediana "4" para o nível de maturidade 3 , o fato de não alcançarem esta mediana em todas as perguntas, fez com que, caracterizada a não satisfação de todas as metas para o nível da maturidade, as organizações não alcançassem um nível de maturidade do processo de desenvolvimento de produtos superior a 2 .

Como pôde ser observado na Tabela 2, as questões 12 - referente ao incentivo, reconhecimento e/ou premiação 
Tabela 5. Cálculo de tendência central e dispersão no Nível de Maturidade 2.

\begin{tabular}{|c|c|c|}
\hline \multicolumn{3}{|c|}{ Distribuição das Pontuações na Questão } \\
\hline Organização & "automóveis" & "caminhões" \\
\hline Mediana & 4 & 4 \\
\hline Desvio médio & 0,665 & 0,643 \\
\hline $\begin{array}{l}\text { Escore mínimo para } \\
\text { Nível de Maturidade }\end{array}$ & 4 & 4 \\
\hline Intervalo interquartil & 1 & 1 \\
\hline
\end{tabular}

Fonte: elaboração própria.

Tabela 6. Compilação das pontuações referentes às perguntas do Nível de Maturidade 2.

\begin{tabular}{lcc}
\hline \multirow{2}{*}{ Respostas } & \multicolumn{2}{c}{ Organização } \\
& "automóveis" & "caminhões" \\
\hline$>4$ & 62 & 63 \\
$<4(\mathrm{x})$ & 36 & 29 \\
$=4$ & 63 & 76 \\
$\Sigma[<4 ;>4](\mathrm{n})$ & 98 & 92 \\
\hline
\end{tabular}

Fonte: elaboração própria.

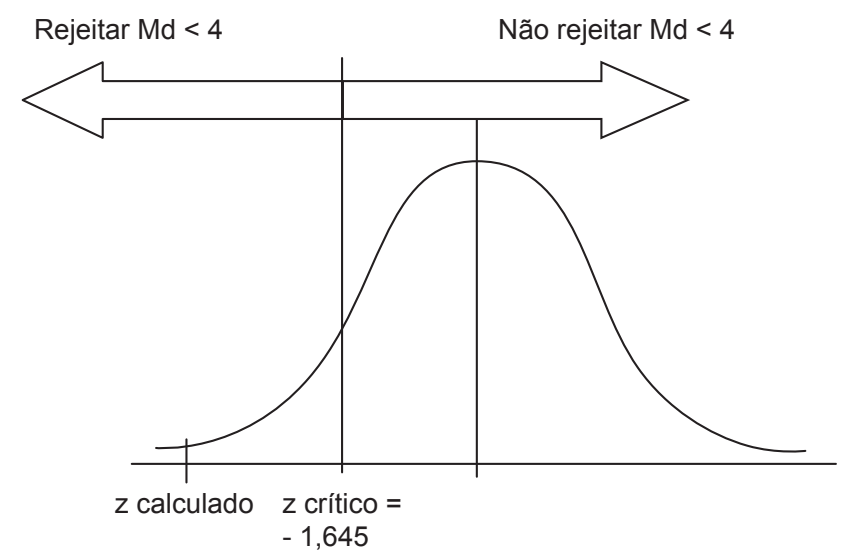

Figura 2. Teste da mediana. Fonte: adaptado de Triola (1999).

da integração (no segmento caminhões - CA) - e 17 - referente ao envolvimento do cliente na validação do atendimento aos requerimentos (ambos os segmentos - CA e AU) - apresentaram o valor da mediana inferior a quatro. Tais itens podem ser considerados pontos prioritários de melhoria para as organizações, com o objetivo de ampliar sua maturidade nos processos de desenvolvimentos de novos produtos.

Com base na premissa listada anteriormente - a metodologia do CMMI é válida para mensuração do nível de maturidade do processo de desenvolvimento de produtos - e nos resultados da pesquisa executada, podem-se estabelecer as seguintes análises quanto ao problema da pesquisa, hipóteses utilizadas e conclusões:

\subsubsection{Solução do problema}

O problema formulado foi: Qual o nível de maturidade dos métodos de desenvolvimento dos produtos utilizados pelas montadoras na região Sul Fluminense?

Segundo a visão dos executivos das organizações, baseado nos critérios do $C M M I$ :

- Todos os projetos da organização asseguram que os requerimentos, produtos e serviços são gerenciados e que os processos são planejados, executados, medidos e controlados;

- O status dos produtos e serviços é visível para a gerência em pontos específicos (marcos referenciais);

- A disciplina de processo ajuda a assegurar que as práticas existentes são mantidas durante os momentos de crise, com os projetos executados e gerenciados conforme os planos documentados;

- Compromissos são estabelecidos entre as partes interessadas (stakeholders) conforme a necessidade, sendo os produtos revistos por estes, para validação do atendimento de seus requerimentos, padrões e objetivos;

- A capabilidade do processo pode ser resumida como sendo disciplinada porque o planejamento e o acompanhamento do projeto são estáveis e os sucessos mais recentes podem ser repetidos; e

- Os processos do projeto estão sob o controle efetivo do sistema de gestão de projeto, seguindo os planos estabelecidos.

\subsubsection{Verificação das hipóteses e questões-chave}

A metodologia aplicada baseou-se no teste de falseabilidade das hipóteses levantadas, por meio do método da hipótese nula, ou seja, pela aplicação de um teste estatístico adequado à natureza das variáveis e da amostra analisada, de forma a verificar-se o grau de significância dos resultados obtidos.

Foram analisados os critérios referentes às práticas representativas de um método estruturado de desenvolvimento de produtos para ambas as organizações. Desta forma, foi avaliada a hipótese em foco, segundo a visão de gerentes e supervisores do corpo técnico de duas montadoras da região Sul Fluminense e, a partir dos resultados coletados no campo, tabulados e tratados de forma estatística adequada. Pôde-se então analisar, validando total ou parcialmente ou refutando, a hipótese levantada e responder às questões-chave associadas.

Hipótese I - Em busca do posicionamento estratégico e manutenção de sua competitividade, as empresas da indústria automotiva mantêm um método estruturado para desenvolvimento de produtos, o qual tem a potencialidade de garantir a aceitação dos consumidores e o 
fluxo financeiro advindo do sucesso comercial dos produtos.

Pelos resultados obtidos, pôde-se concluir que a Hipótese I foi considerada parcialmente plausível, pois em ambas as organizações os respondentes indicaram a existência de um processo de desenvolvimento de produtos, o qual é planejado, executado, medido e controlado e as práticas existentes são mantidas, mesmo nos momentos de crise, podendo repetir a experiência para novos projetos. No entanto, o processo existente não pôde ser caracterizado como tendo os objetivos da organização baseados neste, nem operar dentro de limites estabelecidos ou ser inovado na busca de oportunidades, características estas que garantiriam a aceitação dos consumidores e o fluxo financeiro advindo do sucesso comercial dos produtos desenvolvidos pelo método existente.

Questões-chave:

1) O processo de desenvolvimento de produtos é planejado, executado, medido e controlado e as práticas existentes são mantidas, mesmo nos momentos de crise, podendo repetir a experiência para novos projetos?

Resposta: Sim, pois os respondentes de ambas as organizações indicaram a existência de tais características nos processos existentes de desenvolvimento de produtos em suas organizações.

2) A organização possui um processo de desenvolvimento de produtos bem caracterizado e compreendido, sendo descrito na forma de padrões, procedimentos, ferramentas e métodos, com objetivos baseados nestes e utilizados em todos os processos de desenvolvimento de produtos?

Resposta: Não, os respondentes de ambas as organizações indicaram a existência de lacunas neste aspecto, especificamente o não envolvimento do cliente para validação da verificação de atendimento de requerimentos - para componentes, processos e/ou produto final. Em uma das organizações detectou-se também uma lacuna no que tange ao incentivo, ao reconhecimento e/ou à premiação da integração e colaboração dos envolvidos no desenvolvimento de novos produtos.

3) O processo de desenvolvimento de produtos da organização, já padronizado, é medido e opera dentro dos limites estabelecidos?
Resposta: Não, os respondentes de ambas as organizações indicaram a não satisfação de todas as metas referentes à questão $n^{\circ} 2$, o que caracteriza não haver sustentação para as metas avaliadas nessa questão.

4) A organização trabalha com inovação do processo de desenvolvimento de produtos, identificando as oportunidades de aperfeiçoamento, fortalecendo o processo de maneira pró-ativa e objetivando a prevenção de falhas?

Resposta: Não, conforme resposta da questão anterior.

\section{Conclusões e recomendações}

Pode-se, a partir dos resultados encontrados, concluir que ambas as organizações mostraram utilizar um método estruturado para desenvolvimento de seus produtos, o qual, no entanto, tem campo suficiente - no entendimento dos próprios executivos - de tornar-se mais completo, abrangente e potente para alavancar os resultados de mercado e financeiros da própria organização. Para tanto, recomenda-se a ação sobre os aspectos de integração e colaboração entre os envolvidos no desenvolvimento de novos produtos (no segmento de caminhões e ônibus) e no envolvimento do cliente na verificação de componentes, processos e produto final (ambas as organizações), pontos para melhoria no processo, segundo resultados da presente pesquisa (vide Tabela 2). A eficácia das ações a serem implementadas pode ser verificada em estudo futuro e, como refinamento do método, seria conveniente que se ampliasse o total de pesquisados e que a avaliação dos resultados passasse, além dos métodos e técnicas utilizados neste estudo, pelo crivo de avaliação da lógica paraconsistente (Costa et al., 1999; Carvalho, 2002) e/ou lógica fuzzy, inclusive ponderando as respostas com base no nível de envolvimento e influência das pessoas sobre o processo, de forma a dar maior respaldo e robustez às análises.

\section{Agradecimentos}

Os autores agradecem os comentários e críticas dos revisores e da Editoria, os quais contribuíram substancialmente para a melhoria do artigo. 


\section{APÊNDICE}

\section{Justificativa do Emprego das Questões-Chave para a Hipótese I}

Em busca do posicionamento estratégico e manutenção de sua competitividade, as empresas da indústria automotiva mantêm um método estruturado para desenvolvimento de produtos, o qual tem a potencialidade de garantir a aceitação dos consumidores e o fluxo financeiro advindo do sucesso comercial dos produtos.

\begin{tabular}{l} 
Questões-Chave \\
\hline 1. O processo de desenvolvimento de produto \\
é planejado, executado, medido e controlado \\
e as práticas existentes são mantidas, mesmo \\
nos momentos de crise, podendo repetir a ex- \\
periência para novos projetos?
\end{tabular}

2. A organização possui um processo de desenvolvimento de produtos bem caracterizado e compreendido, sendo descrito na forma de padrões, procedimentos, ferramentas e métodos, com objetivos baseados nestes e utilizado em todos os projetos de desenvolvimento de produtos?

3. O processo de desenvolvimento de produtos da organização, já padronizado, é medido e opera dentro dos limites estabelecidos?
Justificativa da Questão para Teste da Hipótese

O objetivo desta questão é identificar, por meio da aplicação de perguntas específicas no questionário de pesquisa, se o processo de desenvolvimento de produtos está no Nível 2 do CMMI, por meio das seguintes características: requerimentos, produtos e serviços gerenciados, processos executados, medidos e controlados, status de produtos e serviços visíveis para a gerência, compromissos estabelecidos entre stakeholders (perguntas de 1 a 7 do questionário).

O objetivo desta questão é identificar, por meio da aplicação de perguntas específicas no questionário de pesquisa, se o processo de desenvolvimento de produtos está no Nível 3 do CMMI, por meio das seguintes características: processos caracterizados, compreendidos, documentados e estabelecidos, necessidades de treinamento identificadas, partes envolvidas integradas e riscos avaliados (perguntas de 8 a 19 do questionário).

O objetivo desta questão é identificar, por meio da aplicação de perguntas específicas no questionário de pesquisa, se o processo de desenvolvimento de produtos está no Nível 4 do CMMI, por meio das seguintes características: capabilidade de processo previsível, medições de qualidade e performance incorporadas em repositórios, controle de produtos e processos, com redução da variação de desempenho (perguntas de 20 a 26 do questionário).

4. A organização trabalha com inovação do O objetivo desta questão é identificar, por meio da aplicação de perguntas específicas processo de desenvolvimento de produtos, no questionário de pesquisa, se o processo de desenvolvimento de produtos está no Níidentificando as oportunidades de aperfeiço- vel 5 do CMMI, por meio das seguintes características: os processos são estabilizados e amento, fortalecendo o processo de maneira gerenciados de forma a prover a melhoria contínua de desempenho, com base no entendipró-ativa e objetivando a prevenção de falhas? nhecimentos e lições aprendidas são continuamente disseminadas para toda a organização (perguntas de 25 a 27 do questionário).

Apêndice - Justificativa das Questões para Teste da Hipótese I. Fonte: elaboração própria.

\section{Referências Bibliográficas}

ALVAREZ, R.; PROENÇA, A.; ANDÉREZ, D. Rio automotivo: elementos da realidade e perspectivas de desenvolvimento. Rio de Janeiro: SEBRAE, 2002.

ASSOCIAÇÃO NACIONAL DOS FABRICANTES DE VEÍCULOS AUTOMOTIVOS - ANFAVEA. Anuário da indústria automobilística brasileira. São Paulo, 2005. Disponível em <http://www.anfavea.com.br>. Acesso em: 29 junho 2005.

ARBIX, G.; VEIGA, J. A distribuição de veículos sob fogo cruzado: em busca de um novo equilíbrio de poder no setor automotivo. São Paulo: Federação Nacional da Distribuição de Veículos Automotores, 2003. Disponível em <http://www.fenabrave.org.br>. Acesso em: 4 setembro 2004.

BAXTER, M. Projeto de produto: guia prático para o design de novos produtos. 2. ed. São Paulo: Edgard Blüncher, 2003.
CARVALHO, F. Lógica paraconsistente aplicada em tomadas de decisão. São Paulo: Aleph, 2002.

CLARK, K.; FUJIMOTO, T. Product development performance: strategy, organization, and management in the world auto industry. Boston: Harvard Business School Press, 1991.

CLARK, K.; WHEELWRIGHT, S. Managing new product and process development: text and cases. New York: Free Press, 1992.

COOPER, D.; SCHINDLER, P. Métodos de pesquisa em administração. 7. ed. Porto Alegre: Bookman, 2003.

CORREA, C. As dificuldades de quem produz carros. Revista Exame, São Paulo, v. 38, n. 19, p. 92-97, set. 2004.

COSTA, N. et al. Lógica paraconsistente aplicada. São Paulo: Atlas, 1999. 
COSTA JÚNIOR, A.; SILVA, C. Os fatores de fracasso no desenvolvimento de produtos: um estudo de caso em uma pequena empresa de alta tecnologia. In: CONGRESSO BRASILEIRO DE GESTÃO E DESENVOLVIMENTO DE PRODUTOS, 4, Gramado, 2003. Anais... Gramado: IGDP, 2003. p. 1-8.

CRAWFORD, M. New products management. Maidenhead: McGraw Hill Europe, 1997.

CROSBY, P. Quality is free. New York: McGraw-Hill, 1979.

DANIEL, R. Management Information Crisis. Harvard Business Review, Watertown, v. 6, p. 111-121, sep-oct. 1961.

DIAS, A.; SALERNO, M. Produto mundial, engenharia brasileira: integração de subsidiárias no desenvolvimento de produtos globais na indústria automobilística. São Paulo: Universidade de São Paulo, 2003. 68 p. (Boletim Técnico da Escola Politécnica da USP, n. 169).

GANTEWERKER, S.; MANOSKI, P. The library, not the lab: why it's important to do your homework before hands-on product development work begins. Food Processing, Itasca, v. 64, n. 9, p. 40-43, dec. 2003.

GOLDENSON, D.; GIBSON, D. Demonstrating the impact and benefit of CMMI: an update and preliminary results. Pittsburgh: Software Engineering Institute, Carnegie Mellon University, 2003. 34 p. (Special Report CMU/SEI-2003-SR-009).

GRUENWALD, G. Como desenvolver e lançar um produto novo no mercado. São Paulo: Makron, 1994.

HATCH, T.; URBAN, G. New products: minimizing risks and maximizing creativity. Cambridge: Massachusetts Institute of Technology, Alfred P. Sloan School of Management, 1974. 28 p. (Working Paper n. 752-74).

HISRICH, R.; PETERS, M. Marketing a new product: its planning, development, and control. Menlo Park: Benjamin Cummings, 1978.

HOEL, P. Estatística matemática. 4. ed. Rio de Janeiro: Guanabara Dois, 1984.

HUMPHREY, W. Characterizing the software process: a maturity framework. Pittsburgh: Software Engineering Institute, 1987. 24 p. (Report CMU/SEI-87-TR-11, ADA182895).

KERZNER, H. Gestão de projetos: as melhores práticas. Porto Alegre: Bookman, 2002.

KOTLER, P.; ARMSTRONG, G. Princípios de marketing. Rio de Janeiro: LTC, 1999.

LEVIN, J. Estatística aplicada a ciências humanas. 2. ed. São Paulo: Habras, 1987.
MATTAR, F. Pesquisa de marketing. São Paulo: Atlas, 1996. v. 2.

MATTAR, F.; SANTOS, D. Gerência de produtos: como tornar seu produto um sucesso. 2. ed. São Paulo: Atlas, 2003.

MUNDIM, A. et al. Aplicando o cenário de desenvolvimento de produtos em um caso prático de capacitação profissional. Revista Gestão \& Produção. São Carlos, v. 9, n. 1, p. 1-16.

NORTON, D.; KAPLAN, R. Estratégia em ação: balanced scorecard. Rio de Janeiro: Campus, 1997.

ORGANISATION INTERNATIONALE DES CONSTRUCTEURS D'AUTOMOBILES - OICA. World motor vehicle production by country. Disponível em $<$ http://www.oica.net/htdocs/Main.htm>. Acesso em: 4 set. 2004.

OLIVEIRA, W. Modelos de maturidade: visão geral. Revista MundoPM - Project Management, Porto Alegre, v. 6, p. 6-11, dez-jan. 2006.

PROJECT MANAGEMENT INSTITUTE - PMI. Organizational project management maturity model. Newtown Square: PMI, 2003.

QUINTELLA, H. Inovação, estratégia e gestão do produto: gestão total do produto. Rio de Janeiro: Tamas, 2000. v. 1.

QUINTELLA, H.; ROCHA, H. Medindo o nível de maturidade dos processos de desenvolvimento de produtos nas montadoras de veículos com o CMMI. Revista MundoPM - Project Management, Porto Alegre, v. 6, p. 20-27, dez-jan 2006.

QUINTELLA, H.; ROCHA, H.; ALVES, M. Projetos de veículos automotores: fatores críticos de sucesso. Revista Produção, São Paulo, v. 15, n. 3, p. 334-346, set-dez. 2005.

QUINTELLA, H.; ROCHA, H.; MOTTA, W. Avaliação do nível de maturidade dos processos de desenvolvimento de produtos na indústria automotiva do sul fluminense com base nos critérios do CMMI. Relatórios de pesquisa em engenharia de produção, Niterói, v. 5, n. 13, p. 1-15, jul. 2005.

ROCHA, H. Fatores críticos de sucesso do start up de veículos e a qualidade (CMMI) do desenvolvimento de produtos no Sul Fluminense. 2005. 353 f. Dissertação (Mestrado em Sistemas de Gestão) - Universidade Federal Fluminense, Niterói, 2005.

ROCKART, J. A new approach to defining the chief executive's information needs. Cambridge: Center for Information Systems Research, Sloan School of Management, Massachusetts Institute of Technology, 1978. 32 p. (Working Paper n. 37). 
ROCKART, J. Chief executives define their own data needs. Harvard Business Review, Watertown, v. 57, p. 81-83, mar-apr. 1979.

ROZENFELD, H.; AMARAL, D. Proposta de uma tipologia de processos de desenvolvimento de produto visando a construção de modelos de referência. In: CONGRESSO BRASILEIRO DE GESTÃO E DESENVOLVIMENTO DE PRODUTOS. Belo Horizonte, 1999. Anais... Belo Horizonte: IGDP, 1999. p. 1-10.

ROZENFELD, H.; SCALICE, R.; AMARAL, D. Proposta de um método de maturidade para o processo de desenvolvimento de produtos. In: CONGRESSO BRASILEIRO DE GESTÃO DE DESENVOLVIMENTO DE PRODUTOS, 5, Curitiba, 2005. Anais... Curitiba: IGDP, 2005. p. $1-8$.

SCHIBUOLA, T. Eles Desenham as Máquinas. Exame Primeira Pessoa. Separata de: Revista Exame, São Paulo, v. 832, n. 24, p. 60-64, 2004.

SOFTWARE ENGINEERING INSTITUTE - SEI. Capability maturity model integration for systems engineering, software engineering, and integrated product and process development, version 1.1. Pittsburgh: Carnegie Mellon, 2001a. 732 p. (Report CMMI-SE/SW/ IPPD).

Standard CMMI appraisal method for process improvement (SCAMPI), version 1.1. Pittsburgh: Carnegie Mellon, 2001b. 162 p. (Method Definition Document CMMI-SEI-2001-HB-001).

. CMMI general information. Pittsburgh: Carnegie Mellon, 2004. Disponível em <http://www.sei.cmu.edu/ cmmi/general/general.html>. Acesso em: 10 agosto 2004.
SEMENIK, R.; BASSOMY, G. Princípios de marketing: uma perspectiva global. São Paulo: Makron, 1996.

SHULMAN, R. Recovery and the new product paradox. Brandweek, North Hollywood, v. 44, n. 25, p. 20, Jun. 2003.

SIEGEL, S. Estatística não-paramétrica para as ciências do comportamento. São Paulo: McGraw-Hill, 1981.

SILVA, C. Método para avaliação do desempenho do processo de desenvolvimento de produtos. 2001, 187 f. Tese (Doutorado em Engenharia de Produção) - Programa de Pós-graduação em Engenharia de Produção, Universidade Federal de Santa Catarina, Florianópolis, 2001.

SOFTWARE PRODUCTIVITY CONSORTIUM. IPDCMM. Herndon, 2004. Disponível em <http://www.software.org/quagmire/descriptions/ipdcmm.asp $>$. Acesso em: 01 julho 2004 .

SORIMA NETO, J.; GRINBAUM, R. Carro à Brasileira. Época Negócios. Separata de: Revista Época, São Paulo, n. 334, p.12-15, out. 2004.

SOTILLE, M. PMBOK \& CMM + CMMI. Porto Alegre, 2003. Disponível em http://www.pmtech.com.br. Acesso em: 15 fev. 2004.

TRIOLA, M. Introdução à estatística. 7. ed. Rio de Janeiro: LTC, 1999.

URBAN, G.; HAUSER, J. Design and marketing of new products. New Jersey: Prenctice Hall, 1993.

WILSON, C.; KENNEDY, M.; TRAMMELL, C. Superior product development: managing the process for innovative products. Cambridge: Balckwell, 1996.

\title{
EVALUATION OF THE MATURITY OF THE AUTOMOTIVE VEHICLE DEVELOPMENT PROCESS
}

\begin{abstract}
This research purported to evaluate the level of capability maturity of organizations in their product development processes, based on the CMMI (Capability Maturity Model Integration) criteria. The theoretical reference used here was the capability maturity model for integrated product and process development of Carnegie Mellon University's Software Engineering Institute -SEI. Based on the literature about the subject and on a research questionnaire applied at two automaker plants in southern Rio de Janeiro state, Brazil, the study revealed the existence of a planned, executed, measured and controlled product development process. It was also found that the existing practices are maintained even during periods of crises and that the experience can be replicated in new projects.
\end{abstract}

Keywords: automotive vehicles, product development, CMMI. 